\title{
Algunos cuestionamientos a la enseñanza de Ingeniería Industrial en Colombia
}

Some quiestions for industrial engineering education in Colombia

Quelques questionnements sur l'enseignement de l'ingénierie industrielle en Colombie

Luisa F. Rodríguez Valbuena luisafrv@gmail.com Universidad Autónoma de Colombia Bogotá - Colombia.

Candidata del Doctorado Interinstitucional en Educación

por la Universidad Distrital

Francisco José de Caldas.

Grupo de investigación

Formación de Educadores, Docente Adjunta de la Facultad de Ingeniería en la Universidad

Autónoma de Colombia.

Artículo de reflexión Según Clasificación Colciencias

Fecha de recepción: $12 / 03 / 2012$

Fecha de corrección: $27 / 06 / 2012$

Fecha de aprobación: 05/12/2012

\section{Resumen}

En el presente trabajo se pretende hacer un análisis a los procesos de configuración del saber enseñable en ingeniería industrial en Colombia, que tanto en sus inicios como actualmente, correspondieron con los enunciados de modernización impuestos y adoptados por nuestras facultades de ingeniería, sin mayor reflexión, los cuales en este momento son ampliamente cuestionados en todo el mundo, por servir como instrumentos para el extractivismo de nuestros recursos naturales y el empobrecimiento, despojo y desalojo de nuestras poblaciones. Se intenta con este trabajo entregar algunos argumentos iniciales que alienten unas nuevas dinámicas y formas de circulación del saber, diferentes a las que dieron origen a este campo en Colombia. De la mano de algunos de los más reconocidos pensadores del momento, a la luz de la teoría de campos de Bourdieu y las teorías que sobre el poder propone Michael Foucault, se intentará responder algunas de las preguntas que trasnochan a más de uno, en este el oficio de procurar ser maestros.

Palabras clave: educación en ingeniería industrial, teoría de campos de bourdieu, Colombia, modernización y desarrollo. 


\section{Some quiestions for industrial engineering education in Colombia}

Algunos cuestionamientos a la enseñanza de Ingeniería Industrial en Colombia

Quelques questionnements sur l'enseignement de l’ingénierie industrielle en Colombie

\section{Abstract}

Herein, we sought to analyze the configuration of teachable learning processes in industrial engineering in Colombia, both in its inception and currently, corresponded with modernization statements, adopted and imposed by our engineering schools, without further thought, which are now widely questioned worldwide because they serve as instruments for the extractivism of our natural resources and impoverishment, dispossession and eviction of our peoples..This work seeks to provide some initial arguments to encourage new dynamic forms of circulation of knowledge different from those that gave rise to this field in our country. With the help of some of the most renowned thinkers of these times, through the light of Bourdieu's field's theory and power theories that Michael Foucault proposed we try to answer these questions that make us ponder on the task of trying to be teachers.

Keywords: industrial engineering education, fields theory by bourdieu, Colombia, modernization and development.
Quelques questionnements sur l’enseignement de l' ingénierie industrielle en Colombie

Algunos cuestionamientos a la enseñanza de Ingeniería Industrial en Colombia

Some quiestions for industrial engineering education in Colombia

\section{Résumée}

Ce travail prétend faire une analyse des processus de configuration du savoir enseignable en Ingénierie Industrielle en Colombie. Ces processus sont alignés, tant dans ses débuts comme actuellement, avec les énoncés de modernisation imposés et adoptés par les facultés d`ingénierie, qui sont en ce moment amplement questionnés dans le monde entier puisqu'ils servent d'instruments pour l'extractivisme de nos ressources naturelles et l'appauvrissement, dépossession et expulsion de nos populations. On cherche avec ce travail présenter quelques premiers arguments qui encouragent de nouvelles dynamiques et formes de circulation du savoir, différentes de celles qui ont donné origine à ce domaine en Colombie. De la main de quelques uns des plus reconnus penseurs du moment, à la lumière de la théorie des champs de Bourdieu et les théories sur le pouvoir de Michel Foucault, on essayera de répondre aux questions qui font passer des nuits blanches à plus d'une personne, dans cet office d'être enseignant.

Mots clef: éducation en ingénierie industrielle, théorie des champs de bourdieu, Colombie, modernisation et développement. 


\section{Algunos cuestionamientos a la enseñanza de Ingeniería Industrial en Colombia}

\section{Introducción}

La ingeniería industrial al igual que la administración de empresas como disciplinas encargadas de la gestión, la planeación y el control de los recursos en una organización, que al estar fundamentadas en los principios de racionalización económica, tendrían sus orígenes en la implementación del capitalismo industrial en nuestro contexto y estarían asociadas con el momento de la instalación del discurso del desarrollo y de modernización en la segunda mitad del siglo XX. El presente trabajo es una invitación a hacer una lectura diferente de los acontecimientos que trajo consigo el desarrollo y la idea de progreso que fue insertada de inmediato en las mentes de quienes se reconocieron como subdesarrollados en ese momento, sobre todo en las áreas técnicas y de gestión como la ingeniería y la administración.

Jacques Aprile ${ }^{1}$ (2012) en la conferencia inaugural presentada en el Congreso Colombiano de Historia titulada "Memorias del despojo y del destierro", describe con la ayuda de un mapa donde ubica todos y cada uno de los puntos que menciona, encontrando que en su totalidad, el territorio colombiano ha sido tocado por las prácticas de desalojo y despojo. Describe cómo durante la Conquista, la fundación de las ciudades fue una exigencia de la corona para otorgar títulos y a esto se debieron las tantas fundaciones de la época, como la liquidación de los pueblos indígenas, propiciada por las compañías extranjeras para la explotación y la colonización antioqueña; la desaparición de tribus del alto Atrato en 1690, por la explotación minera y esclavista. Luego en 1850 expulsan los mineros mazamorreros, las compañías mineras, al tiempo que Ernesto Cortizos y Aquileo Parra buscan apoyo para eliminar indígenas en el sur de Bucaramanga. Más tarde en 1905, en la zona de Chucurrí, sur de Barrancabermeja, el General presidente Rafael Reyes regala a Virgilio Barco y a Roberto de Mares, la zona, quienes se valen de las fuerzas estatales para limpiarla y luego a través de los contactos con compañías extranjeras se dedican a la explotación del petróleo en la región. La extracción minera de oro en Timbiquí en 1926, trabajo de
Tirado (1976)y el proceso de explotación de las bananeras. El conflicto de los Caicedo en el Valle y la fundación de Caicedonia. El trabajo de Rivas (1899) que describe cómo en 1850 expulsan a los indígenas de la zona del Sumapaz, Carmen de Apicalá, para cultivar café, quina y añil. Tras el lema de "Civilizar a los salvajes", difundido por las órdenes religiosas, son expulsados indígenas en Nariño, proceso documentado en los trabajos de Bonilla (1982).

Desde la conquista hasta hoy, los procesos territoriales, el destierro y el conflicto han estado ligados con la historia urbana, en la que el antagonismo de clases en la urbe y la violación de todo tipo están estrechamente relacionados con la apropiación privada del espacio. Estos han sido el detonante de conflictos y del desarrollo de fuerzas productivas por el ensanche de los medios de producción a partir del desalojo de poblaciones intactas, desalojos dirigidos por ejércitos estatales o privados paraestatales. Luego casi se podría afirmar, escuchando a Aprile (2012), que el corazón de la historia colombiana, es, ha sido y seguirá siendo, la ambición creciente por la posesión territorial. Cualquier parecido con los hechos actuales de despojo y desplazamiento vividos por los pueblos indígenas en Toribio (Cauca), por otros tantos pueblos de la Serranía del Baudó en el Chocó, de la altillanura en la Orinoquia, por la explotación extensiva de coltán o por la inundación del Quimbo en el Huila, seguramente no son pura coincidencia.

Reflexiones como esa nos hacen pensar en el papel que ha jugado la industria en todo este engranaje de despojo y desolación de nuestros ancestros lejanos o cercanos, en la construcción de la sociedad en que hoy vivimos y en el sistema social y económico al que le servimos. Aprile (2012) afirmó también en su conferencia, que la industria ha sido la gran receptora de las fuerzas productivas generadas por los desplazamientos y despojos del campo hacia las grandes urbes, prácticas estas adoptadas por los ejércitos estatales o paraestatales en procura de la protección de grandes extensiones dedicadas a la extracción y explotación extensiva de los recursos en

1. Jacques Aprile Gniset es considerado el historiador de la ciudad colombiana por sus investigaciones en todo el país. Él se definió de la siguiente manera: "No soy ni historiador, ni antropólogo... soy urbanista, un hombre que trabaja con el espacio y lo transforma en vida". Jacques no concibe la historia sin suelo, ucho menos con la importancia que tiene este, en la configuración de la historia de Colombia. 
nuestro suelo. De esta manera, la industria en nuestros países se convierte en ese lugar de encierro, control y disciplinamiento, en la gran y costosa guardería de las poblaciones desplazadas. Y la universidad al igual que la industria, en el lugar de adiestramiento para el trabajo, encargada de la docilización de la población, especialmente en las áreas técnicas como la ingeniería industrial.

\section{Modernidad, modernización y crecimiento económico}

Intentando pensar en las tensiones o rupturas que pudiesen constituirse en condiciones de posibilidad de la ingeniería industrial en nuestro entorno, surge la idea de modernidad, que de la mano con la noción de racionalidad hace pensar en múltiples acontecimientos: el renacimiento, la ilustración, la consolidación del capitalismo y la aparición de la noción de técnica en el campo económico, son muchos y diversos los teóricos que la abordaron, algunos como Melo (1990) consideran que la modernidad se instauró con la aparición de la idea ilustrada de progreso en las primeras etapas de modernización del siglo XVII, que enfrentó a los "anciens" y los "modernes" en la Francia de finales de siglo, y se constituyó en el principal elemento de racionalidad en la sociedad moderna del siglo XVIII con la aplicación de las ciencias de la naturaleza. Weber (1905) por ejemplo, resalta el valor de la técnica en la exaltación del espíritu capitalista de la Europa de finales del siglo XIX. Marx (1844) define el proceso de acumulación simple como la condición de existencia del capitalismo que caracterizó el mundo moderno. $Y$ Touraine quien presenta la racionalidad instrumental como la base del capitalismo característica de las sociedades industriales, que al estar sustentada en la técnica, acabó con la racionalidad objetiva de las ciencias del renacimiento y la ilustración.

La crisis y la decadencia de la razón objetiva determinan la progresiva separación de cuatro universos culturales: Eros, el consumo, la empresa y la nación, pero estos universos están también relacionados entre sí por la razón instrumental que para decirlo con mayor claridad es la técnica. Lo cual está de acuerdo con la visión de Weber y de Horkheimer. La razón se ha hecho sólo instrumental; la racionalidad de los medios reemplaza la racionalidad orientada hacia los fines. Esto es lo que define a una sociedad industrial que asigna un lugar central a la producción y a la distribución masiva de bienes de consumo y servicios. (Touraine, 2000, p. 146)

La idea de modernidad en América Latina adquiere un nuevo impulso con la instauración del capitalismo como modelo económico preponderante en nuestras sociedades, cuyas repercusiones más contundentes se dieron con la instalación del discurso del desarrollo a mediados del siglo pasado. Algunos autores latinoamericanos como Escobar (1996) enfatizan en la aparición del concepto de desarrollo luego de la segunda posguerra formulado inicialmente en Estados Unidos y Europa y ansiosamente aceptado y mejorado por las élites y gobernantes del Tercer Mundo; a partir de entonces, según él, se dio la transformación total de las culturas y formaciones sociales de tres continentes de acuerdo con los dictámenes de las del llamado Primer Mundo. En el contexto social mundial, según Coombs (1971) la educación adquiere gran importancia. A comienzos de la década de 1950, los sistemas educativos iniciaron un proceso expansivo sin precedentes en la historia humana. A mediados del siglo $X X$, todos los países del mundo sufrieron cambios en su medio ambiente a una velocidad vertiginosa, como consecuencia de simultáneas revoluciones mundiales en la ciencia y la tecnología, la política y la economía, las estructuras demográficas y sociales.

En Colombia autores como Martínez al igual que Escobar ubican el acontecimiento de modernización al tiempo con la introducción del desarrollo y aportan un nuevo elemento de análisis a partir del concepto de expansión educativa como dispositivo de sometimiento y control de la totalidad de la población en tanto que mecanismo de inclusión:

[...]La inclusión significaba control y gestión de la totalidad de la población, y no sólo de unos sectores, para lo cual era necesario construir a través del discurso formas de subjetividad, es decir, lograr que las personas se reconocieran a sí mismas como subdesarrolladas. [...] la expansión educativa se presenta precisamente como la estrategia fundamental que permitía garantizar el ingreso, retención y calificación de la población por parte de la escuela. Desbloquear la escolarización significaba, entonces, incluir el mayor número de sectores sociales a las tareas de la modernización social. (Martínez, 2004, p.49) 
Ambos autores coinciden en afirmar que el naciente orden del capitalismo y la modernidad, inserto en nuestras sociedades luego del discurso del desarrollo, dependían de una política de lucha contra la pobreza más que del poder industrial y tecnológico, cuya intención era no solo crear consumidores sino transformar la sociedad, convirtiendo a los pobres en objetos de conocimiento y administración. La pobreza se asociaba con rasgos como movilidad, vagancia, independencia, frugalidad, promiscuidad, ignorancia y la negativa a aceptar los deberes sociales, a trabajar y a someterse con la lógica de la expansión de las "necesidades". Por consiguiente, la administración de la pobreza exigía la intervención en educación, salud, higiene, moralidad, empleo, la enseñanza de buenos hábitos de asociación, ahorro, crianza de los hijos, y así sucesivamente. El crecimiento económico presuponía la reproducción en los países pobres de las condiciones que caracterizaban a los países capitalistas avanzados, incluyendo la industrialización, la urbanización, la modernización agrícola, la infraestructura, el creciente suministro de servicios sociales y los altos niveles de alfabetismo (Figura 1).

\section{Figura 1. Dispositivos y mecanismos con los que operó el modelo de desarrollo*.}

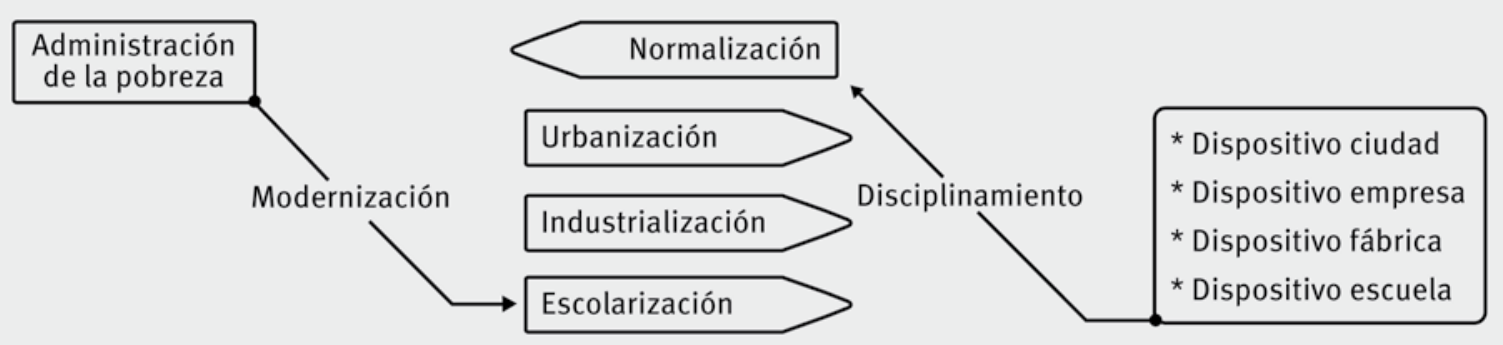

* La modernización para garantizar la efectiva administración de la pobreza se valió de procesos como la escolarización, la industrialización y el urbanismo como mecanismos de disciplinamiento y normalización, mediante el fortalecimiento de nuevos dispositivos de encierro como la fábrica y la empresa o la masificación de otros como la escuela, la universidad y la ciudad, en la nueva sociedad latinoamericana luego de la segunda posguerra.

Fuente: Rodriguez, L. F. (2012).

Al igual que Escobar (1996, p. 109), Corredor (1992, p. 110) y Díez (1995, p.36) afirman que el modelo de desarrollo económico, al privilegiar los fines sobre los medios, no haciendo partícipe a las mayorías, los habitantes del llamado Tercer Mundo, en vías de desarrollo, que constituyen las tres cuartas partes de la humanidad y que han visto ese progreso como algo que existe a expensas de sus recursos naturales, de sus respectivas identidades culturales y sin recibir los beneficios del crecimiento, de la investigación científica y del progreso tecnológico, se encuentra sumergido en una profunda crisis. Escobar (1996) afirma por ejemplo: "La gente no asistía alegre ni por su propia voluntad a las fábricas." Por el contrario, se necesitó de un régimen completo de disciplina y normalización, además de la expulsión de campesinos y siervos de sus tierras y de la creación de una clase proletaria. La economía moderna exigió la reestructuración profunda de los cuerpos, los individuos y las formas sociales.

De esta manera tanto Escobar como Martínez atribuyen a la Teoría del Capital Humano todo el soporte teórico del discurso desarrollista implementado en las sociedades, porque el argu- mento central de esta teoría es que inversiones sistemáticas en educación y salud aumentarían la productividad de la mano de obra, lo cual se traduciría en crecimiento económico, es decir, que en la teoría del crecimiento económico a los dos factores de producción tradicionales, capital y trabajo, se añade un tercero, "el conocimiento". En este momento le fue atribuida a la educación un papel instrumental en el sentido de que se le adscribió la tarea de adecuar la mano de obra en concordancia con los requisitos y necesidades del sector productivo. La educación se orientó entonces hacia la capacitación y el adiestramiento de los recursos humanos en el dominio de las técnicas específicas para aportar al desarrollo de esos sectores.

Los procesos de modernización con la aplicación de la técnica y los principios de racionalidad económica en nuestras sociedades y la instauración de la producción en masa no solo de bienes de consumo sino de todos los servicios asociados con el urbanismo como la educación, salud e infraestructura que demandaban las ciudades recién urbanizadas, hizo necesaria la existencia de la ingeniería industrial como una de las tantas dis- 
ciplinas instaladas en el momento y que buscaban la administración eficiente de la pobreza.

\section{La Ingeniería Industrial como campo de saber}

Entender la ingeniería industrial como campo de saber, implicaría revisar la noción de campo que desde Bourdieu (2001) presupone el estudio de las múltiples y variadas tensiones que atraviesan y configuran una disciplina, en la producción de lo que Foucault (2005)ha llamado "cuerpos dóciles" a través de un régimen completo de regulación y normalización. Para Foucault la principal función de la disciplina es entrenar individuos, hacer "individuos", estos son objeto de su poder a los cuales se aplican modalidades coercitivas específicas. Foucault caracteriza el papel de la disciplina por medio de la normalización. El poder de la normalización impone homogeneidad pero "individualiza al permitir las desviaciones, determinar los niveles, fijar las especialidades y hacer útiles las diferencias ajustándolas entre sí” (Díaz, 1993, p. 213). Entonces, mientras la normalización introduce un sistema de igualdad formal el cual aparentemente, opaca las diferencias individuales, la individualización objetivamente separa y jerarquiza a los individuos.

Durante el mercantilismo europeo Foucault (2005), describe cómo la burguesía industrial favoreció el surgimiento de la prisión para proteger sus propiedades y dispuso sus fábricas de forma tal que sirviesen a la vez como mecanismo de control para la población plebeya y campesina que empleaba, estableciéndose una identidad morfológica del sistema de poder entre las dos instituciones, prisión y fábrica, que al igual que la escuela como formas de encierro se constituyen en los dispositivos de control y disciplinamiento característicos también en el momento de la industrialización en Latinoamérica.

De esta manera las relaciones de poder se consideran ante todo productivas, en la medida que movilizan acciones convenientes para los agentes que pone en juego y para poder propiciarlas, se valen de dispositivos que favorezcan el disciplinamiento y la normalización de la población que pretenden afectar.

En este punto resulta pertinente citar a Atcon (1978, p.21), quien sostiene:

[...] "la universidad le opone tanta resistencia al cambio como cualquier gen a una mutación. Pero una vez que acepta y se asimila una idea nueva, ésta será inmediatamente transmitida a las generaciones siguientes junto con el resto, con lo pasado, con lo verificado. [...] Entonces habremos logrado lo que nos propusimos, sin violencia y dentro de un espíritu genuino de ayuda a los demás" [...].

Por ello al analizar el acontecimiento de la escolarización como mecanismo de disciplinamiento, el efecto individualizador que enmarca la normalización adquiere su verdadero valor al potenciar al individuo como agente dinamizador de cambio dentro del espacio social en el que se encuentra inmerso posicionándolo como diseñador de estrategias que aseguren su estabilidad dentro de la estructura del campo específico de su quehacer.

Para la siguiente afirmación, se tomará de Deleuze (1987, p. 102), quien interpretando a Foucault, sostiene que: "toda relación de fuerzas es una 'relación de poder' [...] la relación de poder no se produce entre dos formas, como el saber".

Por tanto al definir la universidad como el espacio de fuerzas que se establecen entre los agentes de los diferentes campos disciplinares, al aplicar estrategias para adquirir o mantener los diferentes tipos de capitales que les permiten mantener su posición en la estructura del campo (Bourdieu, 2008). Es fácil pensar en el proceso de configuración de la ingeniería industrial como campo de saber a partir del choque entre las fuerzas modernizadoras impuestas con la instauración del discurso del desarrollo y los diferentes agentes responsables de la industrialización del país. Fuerzas modernizadoras como la del urbanismo, la industrialización y la escolarización, pusieron a disposición de los agentes e instituciones que iban creando o afectando a su paso, nuevas discursividades y formas de subjetividad que motivaron diferentes formas de representación de los sujetos involucrados y nuevas formas de circulación del saber.

Deleuze (1987, p. 65) sostiene que "No existe relación de poder sin la constitución correlativa de un campo de saber, ni saber que no suponga y no constituya al mismo tiempo relaciones de poder".

La ingeniería industrial deviene como una de esas nuevas formas de disposición que fue preciso que asumieran los agentes responsables de la industrialización, al hacerse necesaria la aplicación de los principios de racionalidad económica de Taylor (1911) en las recién creadas fábricas, que muy pronto se constituyeron en organizaciones que producían un amplio espectro de bienes y servicios y entonces fueron denotadas con la distinción de empresas, 
convirtiéndose en objeto de saber escolarizable y por tanto en una nueva disciplina.

De este modo Deleuze (1987, p. 52-53) afirma que:

\begin{abstract}
"Una de las ideas esenciales de vigilar y castigar es que las sociedades modernas pueden definirse como sociedades 'disciplinarias'; pero la disciplina no puede identificarse con una institución ni con un aparato, precisamente porque es un tipo de poder, una tecnología, que atraviesa todo tipo de aparatos y de instituciones a fin de unirlos, prolongarlos, hacer que converjan, hacer que se manifiesten de una nueva manera"
\end{abstract}

Los principios de eficiencia y productividad sobre los cuales se fundamenta la racionalidad económica del proceso de industrialización se convirtieron en el principal capital y elemento constitutivo del habitus o dominio de acción propios del ingeniero industrial. El aparato escolar como responsable de capacitar la mayor cantidad de mano de obra de manera eficiente y productiva se convierte en una empresa más, la universidad adquiere nuevas funciones al encargarse de acercar el conocimiento con el mundo del trabajo (Unesco, 1998) y formar en este proceso a los nuevos agentes del campo industrial. Se crean también otras instituciones como los institutos de desarrollo científico y tecnológico (Bustamante, 1996) a partir de la alianza entre los gremios de la producción y el sector académico, proceso esquematizado en la Figura 2. El servicio de la educación en ingeniería junto con sus productos asociados como innovación y tecnología, pasan a ser servicios de consumo masivo en el paradigma de modernidad que caracterizará las sociedades del conocimiento.

Figura 2. Nuevas dinámicas creadas con la aplicación de la teoría del crecimiento económico*

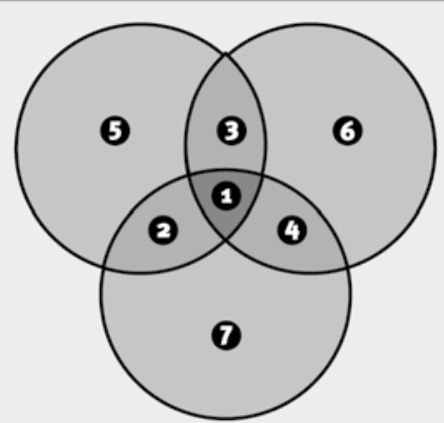
1. CII
2. Universidad
3. Empresa
4. Instituto de desarrollo tecnológico
5. Trabajo
6. Capital
7. Conocimiento

* El integrar el conocimiento garantiza la productividad de la mano de obra, adscribe nuevas funciones a las instituciones ya existentes y crea otras.

Fuente: Rodríguez, L. F. (2012).
A la luz de la teoría de campos de Bourdieu (2001), a partir de la introducción del modelo de modernización empieza a circular como forma de regulación el capital escolar, académico y posteriormente informacional, adicional al laboral y económico que caracterizó las sociedades anteriores. Las diferentes universidades compiten entre ellas por el dominio de los diferentes tipos de capital al igual que las empresas, son creados nuevos emplazamientos como los institutos de desarrollo tecnológico que también compiten con las universidades por el capital intelectual.

El espacio social de la universidad, luego de la posguerra en la nueva sociedad disciplinada, asume la responsabilidad de integrar el conocimiento al mundo productivo para garantizar el progreso científico y tecnológico del país y el campo de la ingeniería industrial (CII) se configura al igual que otras disciplinas, como la administración de empresas, entre el capital, el trabajo y el conocimiento representado por las universidades y todas las instancias de saber. Es decir, como ese nuevo "tipo de poder, de tecnología, que atraviesa todo tipo de aparatos y de instituciones a fin de unirlos, prolongarlos", haciendo que converjan y se manifiesten de una nueva manera, al que se refería Foucault citado por Deleuze (1987), cuando definía la noción de disciplina.

Las agencias internacionales (Banco Mundial, 2000), promueven la competencia entre las universidades por el dominio del capital intelectual en el paradigma de la globalización. Igual que la universidad, la empresa se configura como espacio de fuerzas en el que diferentes agentes ponen a prueba estrategias económicas que garanticen mantener su posición en la estructura generando una organización eficiente y productiva.

Recordando a Bourdieu (2000, p. 95), quien sostiene:

"Las empresas [...] son unidades relativamente autónomas que funcionan también como campos [...] Dividida en organizaciones esencialmente dirigidas hacia la producción, la investigación, el marketing, la financiación, etc., se compone de agentes cuyos intereses específicos están relacionados con cada una de esas organizaciones y de esas funciones [...] Sus estrategias son fruto de la relación entre, por un lado unos intereses y unas disposiciones vinculados a unas posiciones en las relaciones de fuerza en el seno de la empresa [...] la estructura de la relación de fuerza entre los diferentes agentes que forman parte 
de ella es proporcionada a su peso individual en cuanto al volumen de su capital".

Y apoyándose en Touraine (1969, p. 144-145), que al afirmar: "La formación de grandes empresas mecanizadas, el estudio no ya solamente de las máquinas, sino de los talleres, conducen a la concepción de que la eficacia de la empresa depende en gran parte de su eficiencia como organización". Aporta la dimensión de eficiencia al concepto de la nueva organización universidad.

Surgen diferentes tipos de universidades con los procesos de diversificación educativa, de esta manera son creadas universidades pequeñas sin mucho o nulo contacto con el mundo productivo e igualmente surgen empresas pequeñas al margen de la universidad y de la llamada generación de conocimiento e investigación científica (Figura 3).

Figura 3. Estructuración del campo social de la ingeniería industrial.*

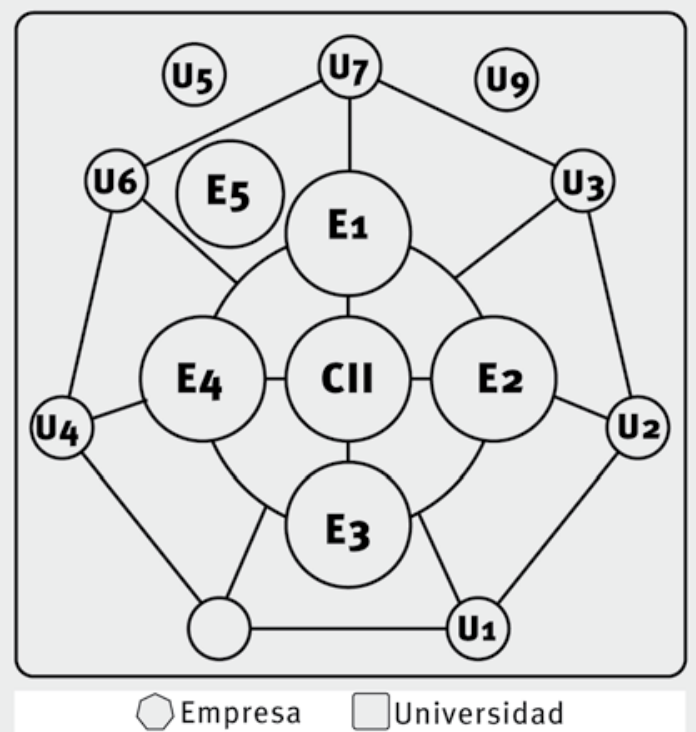

* Las universidades de manera similar que las empresas en el paradigma del desarrollo y el actual de la globalización, compiten entre ellas por el dominio del capital científico y tecnológico. El campo de la ingeniería industrial se configura entre el espacio empresarial y universitario.

\section{Fuente: Rodríguez, L. F. (2012).}

Como antesala a la siguiente afirmación, me valgo de los argumentos de Deleuze (1987, p. 18) quien: "hace del análisis foucaultiano de la subjetividad en sus últimos textos, como un tercer dominio, derivado del poder y el saber, pero independiente, un tercer dominio que es tanto condición de posibilidad del pensar como del resistir."
En los procesos de subjetivación del habitus inculcado durante su disciplinamiento y para garantizar su reproducción, el ingeniero industrial es adiestrado en torno a distinciones como eficiencia, productividad, gestión y calidad, en correspondencia con los enunciados propios de la racionalidad económica impuesta por el capitalismo instaurado en Latinoamérica a partir del discurso del desarrollo de 1949.

Respecto a la noción de enunciado, conviene hacer uso de la distinción propuesta por Deleuze (1987, p. 34):

el enunciado: éste, tiene un "objeto discursivo" que no consiste en modo alguno en un estado de cosas al que hace referencia, sino que deriva, por el contrario, del propio enunciado. Es un objeto derivado que se define precisamente en el límite de las líneas de variación del enunciado como función primitiva.

Estos enunciados conservan una regularidad que se repite incesantemente en los documentos que sobre educación en ingeniería y desarrollo científico y tecnológico se siguen produciendo. $Y$ es que en últimas el objeto discursivo del crecimiento y desarrollo económico esta soportado sobre los mismos principios de racionalidad económica que motivaron el surgimiento de la ingeniería industrial como disciplina de saber, por ello se constituyen en condiciones de existencia del campo y los procesos de subjetivación por parte de los agentes del campo, de estos enunciados se convirtieron en las condiciones de posibilidad de la disciplina en el mundo desarrollado.

Pero de otro lado si la condición de existencia del campo de la ingeniería industrial como disciplina de saber es la posibilidad de construir sujetos en el dominio enunciativo del discurso del desarrollo, existe también la posibilidad de potenciar sujetos para la resistencia de dicho dominio enunciativo. Más cuando dicho dominio enunciativo que se nos fue impuesto, fue creado en el mundo desarrollado a partir de procesos históricos completamente diferentes a los nuestros. La pregunta sería entonces cómo resistir, si de otro lado ya es muy claro que las teorías de crecimiento económico entraron en crisis al desconocer a los directos afectados, no bastaría simplemente con involucrarlos ahora, sino en repensar más bien, los objetos mismos de enunciación: ¿por qué crecimiento económico como condición del desarrollo? ¿son realmente productivos los principios de eficiencia y productividad económica? ¿qué es ser productivo, y para quién, en un momento dado y en otro no? 


\section{El campo de la Ingeniería Industrial en Colombia}

El proceso de configuración de la ingeniería industrial como campo de saber en Colombia, obedeció a la confluencia de varios acontecimientos relacionados con la implementación de las teorías del desarrollo, crecimiento económico y del capital humano a mediados del siglo XX, a cargo de las familias y monopolios consolidados a partir del despojo y desalojo de las tierras que fueron entregadas a compañías extranjeras para la extracción y explotación de los recursos naturales en el país. Este fenómeno sigue sucediendo aún en la actualidad (Figura 4).

Según Quintero (2004, p. 13) desde 1929 se notaba en Colombia el fortalecimiento de la industria, cuando las empresas monopólicas absorbieron las más pequeñas y empezaron a importar la maquinaria requerida para la producción en masa, lo que producía la racionalidad en el trabajo de modo similar al de las primeras revoluciones industriales en el mundo. La inversión extranjera en la industria colombiana a partir de 1940, el desarrollo de la legislación laboral, la aparición de los sectores bancario y financiero y los diferentes elementos que asentaron el capitalismo en Colombia, fueron aspectos muy importantes en la consolidación de las empresas que implicaron una nueva división social del trabajo en el que también se crearon nuevas profesiones, entre ellas la ingeniería industrial y la administración de empresas.

Es importante resaltar en este punto cómo, contrario a lo que sucedió en los llamados "países desarrollados" donde el proceso de modernización determinó una demanda objetiva de formación técnica de mano de obra como resultado de los requerimientos de las fuerzas productivas traducida en una división técnica del trabajo, en nuestra educación, en la que al estar nuestra sociedad regida por la propiedad privada, esta "demanda objetiva", dependió de la división social del trabajo y de la organización de la producción basada en la selección de acuerdo con las capacidades individuales de los aspirantes a ingresar al sistema universitario obteniendo como resultado la producción de mercancías, es decir, relaciones sociales entre sujetos desiguales o sea mercancías de valor diferente y convirtiéndose en el disfraz dado a la demanda objetiva de recursos humanos profesionales (Henao et al.,1975, p.57-59).

La noción de campo que desde Bourdieu (2001, p.34) presupone el estudio de las múltiples y variadas tensiones que atraviesan y configuran la ingeniería industrial, sirve de marco

\section{Figura 4. Configuración del campo de la ingenieria industrial en Colombia.*}

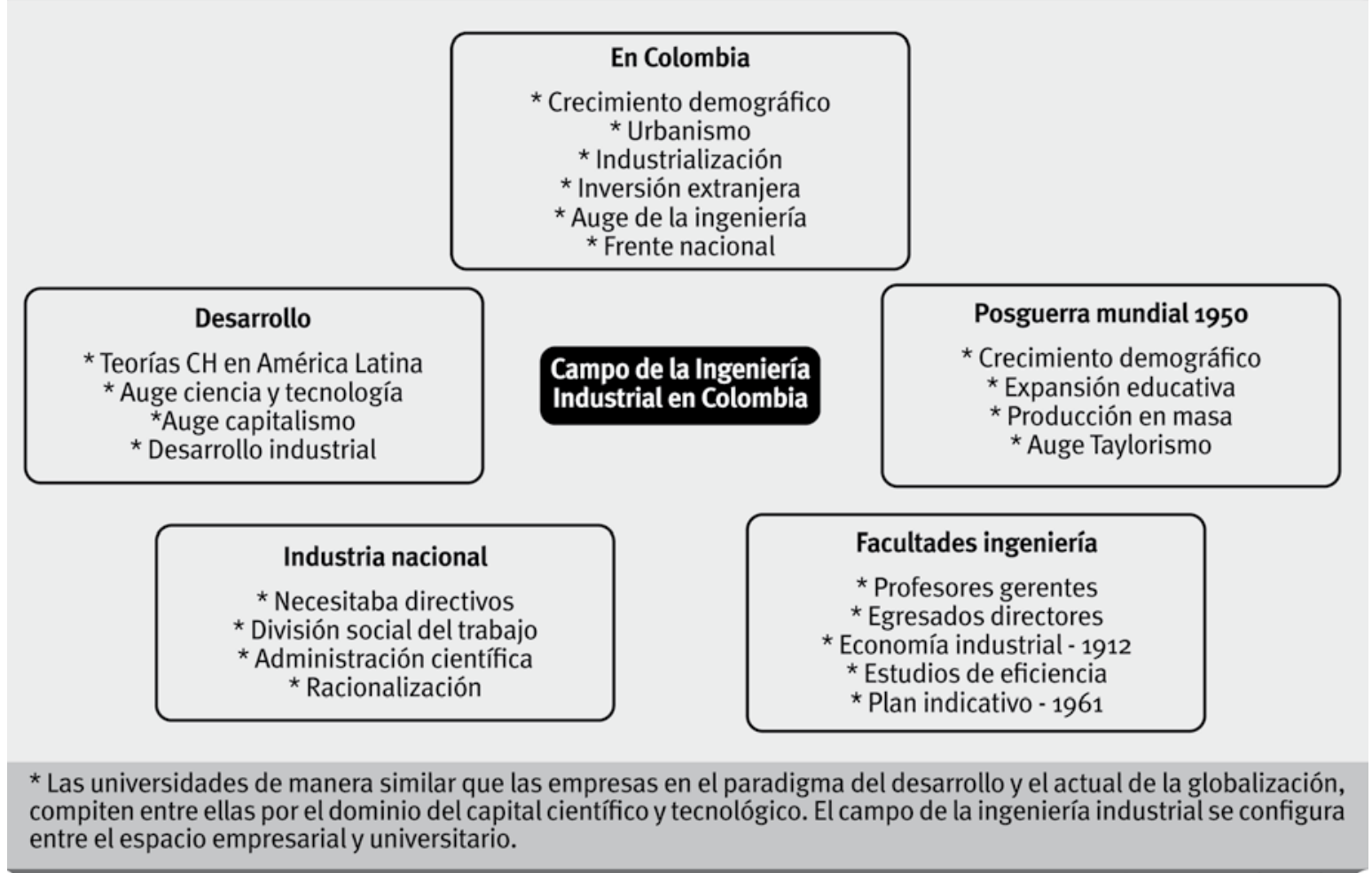


para abordar el problema: Weiss (1994, p. 42), en el estudio que realizó acerca de los orígenes y resultados de los diferentes estilos de liderazgo y gestión en 18 empresas de tres sectores industriales, los alimentos, los minerales no metálicos y metalmecánicas de Bogotá, demuestra como la asimilación y aplicación de las tesis tayloristas y el pensamiento tecnocrático de los ingenieros tuvo características diferentes desde las primeras décadas del siglo XX en Bogotá y en Antioquia. Varios autores citados por Weiss como Savage (1986), Mayor (1990) y Arango (1991), coinciden en afirmar que las relaciones entre los empresarios y los trabajadores en Antioquia se caracterizó porque los planteamientos de Taylor (1911) se adaptaron a las enseñanzas de la iglesia católica: los patronos tenían la responsabilidad de proteger a los obreros y a sus familias. Mientras que los ingenieros bogotanos basaban la relación con sus trabajadores sobre la supuesta superioridad intelectual y la posición social elevada del ingeniero. La formación de los ingenieros en la Universidad Nacional, en Bogotá, por su parte, se orientaba más al cumplimiento de funciones técnicas que de dirección empresarial. Estas concepciones produjeron una asimilación del pensamiento tecnocrático diferente al de los ingenieros antioqueños de la Escuela de Minas, que influía sin duda en sus estilos de dirección empresarial así como en los términos despectivos con que los ingenieros bogotanos se referían a los capataces, obreros y artesanos. Sin embargo, con el tiempo los antioqueños terminaron por adoptar las mismas prácticas de los bogotanos, al unificarse los programas de formación. Según Dávila citado por Weiss (1994), la influencia de las prácticas y la ideología taylorista siguió determinando la mentalidad y las políticas de los ingenieros industriales de las décadas de 1960 y 1970 y aún de 1980 , cuando ya comenzaban a combinarse con algunas teorías sobre la participación con iniciativas como los programas de "desarrollo organizacional".

Paralelo a los cambios de concepción de la ingeniería industrial al interior de las empresas se dio el crecimiento de los programas de formación profesional en las universidades. Las reformas a la educación superior como el Decreto 80 de 1980 (Colombia, 1980) con la misión de ciencia y tecnología, como la Ley 30 de 1992 (Colombia, 1992) concretada en la misión para la modernización de la universidad estatal en 1996, incluyen como factor preponderante para el desarrollo de la educación superior, el énfasis dado a las áreas científicas y tecnológicas, factor este, entre otros que habría propiciado el que la demanda social por la ingeniería industrial se disparara en las últimas décadas de 6.793 solicitudes entre 1974 - 1976 a 17.302 entre 1995 - 1997, datos que hacen, entre otros, según Quintero (2004, p.11) a que la profesión del ingeniero industrial se vea afectada por procesos tanto de masificación como de diferenciación, que determinan que los cargos ocupados por sus profesionales en las empresas, no puedan asociarse sólo con funciones de organización y control del trabajo - tal como sería en sus inicios- sino con una gran variedad de actividades, que van desde la gerencia general hasta tareas propias de trabajadores con calificación técnica, cuyas consecuencias no se manifiestan sólo en un cambio de las cualificaciones sino en la posición social del profesional en las organizaciones y en la sociedad.

Estudios como el de Durán (1989, p. 13-31), acerca del estado de las carreras de ingeniería industrial en Colombia hasta 1989, junto con los estudios del ICFES y ACOFI (1996, p. 5-19), coinciden en afirmar que la estructura curricular de los distintos programas de ingeniería industrial desde que fue fundada en la UIS en 1958, constan de cinco áreas básicas: área de ciencias básicas, área científico tecnológica, área económico administrativa, área socio-humanística y área profesional. Estos trabajos también advierten sobre la necesidad de fomentar la vinculación de las universidades con la industria y con las entidades gubernamentales, así como la necesidad de promover empresas de desarrollo tecnológico mediante incentivos tributarios e ingreso de capitales de riesgo internacionales. También plantean la necesidad de "actualización y modernización de los planes de estudio, que les permita ser competitivos, flexibles, dinámicos y con niveles altos de pertinencia, en concordancia con las necesidades socioeconómicas, culturales, científicas y productivas del país". En el año 1989 existían solo dos programas en funcionamiento que ofrecían el título de Magíster en Ingeniería Industrial: Magíster en Ingeniería Industrial y Sistemas de la Universidad del Valle y el Magíster en Investigación de Operaciones y Estadística de la Universidad Tecnológica de Pereira. La Universidad de los Andes tenía un programa de Magíster en Ingeniería Industrial iniciado en la década de 1970, pero estuvo inactivo durante 1989. Hasta 1989 existían 24 programas aprobados en ingeniería industrial en el país, 9 en Bogotá, 3 en Medellín, 3 en Cali y los demás en otras ciudades. De estas universidades 12 son públicas ( 7 en ciudades importantes, 4 regionales y 1 distrital), y 12 privadas (1 de las privadas es regional). 
En la década de 1990, según Pérez (2004, p. 7-8), el auge de las nuevas tecnologías de información y comunicación, la biotecnología, las tecnologías para la obtención y transformación de nuevos materiales, los sistemas expertos, la inteligencia artificial, la robótica y los nuevos esquemas de manufactura flexible, para citar sólo algunos, que hizo modificar los modelos de producción basados en una demanda estable, en mercados grandes y homogéneos con largos ciclos de producción y de producción en masa, por modelos que por el contrario, suponen demandas fragmentadas, mercados heterogéneos y pequeños, ciclos cortos de productos y esquemas de producción especializada y altamente flexible; se tradujo en una nueva orientación dada a la formación en ingeniería industrial en Colombia, de forma que tuviera en cuenta la innovación, las necesidades de información y el conocimiento tecnológico y organizativo necesario para gestionar adecuadamente los nuevos cambios. Este objetivo, perseguido sin mayores avances hasta nuestros días, que podría evidenciar precisamente el alto grado de imposición, reproducción y dependencia que tiene aún nuestra educación en ingeniería. Ya que en últimas, este objetivo que fue el impulsor de todo el auge tecnológico en el mundo desarrollado, nada ha tenido que ver con los verdaderos motivos que mueven nuestro sector industrial.

\section{Consideraciones finales}

En esta educación en ingeniería tan tradicional y perversamente orientada hacia lo instrumental y operativo muy al margen de la reflexión -porque la reflexión es atribuida casi exclusivamente a las humanidades - según (Mejía, 2009) la educación en ingeniería en Colombia se caracteriza por adoptar una "aproximación puramente estratégica, que podría darle a la ingeniería, un sentido mercenario; es decir, aquél desde el cual los ingenieros utilizan inteligentemente -0 tal vez astutamente- su juicio para tomar las mejores decisiones de diseño de sistemas y lograr un fin que es contratado por el mejor postor, sin preguntarse por el fin en sí mismo. El fin podría ser definido por otros, que tienen el poder para contratar sus servicios".

Visto de esta manera la educación en ingeniería debería contemplar el estudio de las concepciones ideológicas que están detrás de las formas de poder que motivan cualquier implementación de diseños de ingeniería, tanto en sus fines como en sus medios, como de los sistemas sociales sobre los cuales se hace intervención, de los actores involucrados, de sus intereses y propósitos y de las maneras en las que el conocimiento se ha utilizado para legitimar o deslegitimar esos intereses o propósitos.

El intento de pensar unas nuevas relaciones entre capital, trabajo y educación, por ejemplo, a partir de las voces que nunca antes se habían escuchado como las de los indígenas, las de los negros y las de los que piensan diferente, podría resultar en acontecimientos como el que dentro de la formación de los ingenieros, se pudiese incluir, a la par que conceptos como, el de celdas de manufactura flexible o edificios inteligentes, el de mingas u otros conceptos de la racionalidad económica de los pueblos indígenas. Facilitando efectos como, el que una importante fuente de empleo de los ingenieros industriales, a cambio de las anheladas ofertas de las empresas multinacionales, fueran las mingas y los cabildos indígenas, en las que aprendieran y compartieran los saberes ancestrales, sin la mediación de ninguna contraprestación monetaria financiada por ninguna agencia internacional.

Para el caso de los países latinoamericanos, convendría preguntarse cómo y por qué surgió la idea misma de educación en ingeniería, ¿es la educación en ingeniería que hoy se le imparte a los estudiantes en las facultades, basada en el modelo extractivista del "mundo occidental avanzado"? y en esa medida ¿responde a las necesidades de desarrollo de ese mundo y no a las del nuestro? acaso ¿los principios de ingeniería aplicados por nuestros ancestros indígenas insertos en un profundo conocimiento de sí mismos y del cosmos, están incluidas en esos desarrollos del "mundo occidental avanzado"?

\section{Referencias}

Asociación Colombiana de Facultades de Ingeniería (ACOFI). (2001). Historia de las facultades de ingeniería en Colombia. Bogotá, Colombia: ACOFI (pp. 5-24).

Aprile, J. (2012). Conferencia inaugural, memorias del despojo y del destierro. Memorias del XVI Congreso Colombiano de Historia, Neiva, Colombia octubre 8. Asociación Colombiana de Historia.

Arango, L.G. (1991). Mujer, religión e industria. Fabricato 1923-1982. Citado por Weiss, A. (1994). La empresa colombiana entre la tecnocracia y la participación: del taylorismo a la calidad total. Bogotá, Colombia: Departamento de Sociología, Universidad Nacional de Colombia. 
Atcon, R. (1978). El plan básico. La universidad latinoamericana: clave para un enfoque conjunto del desarrollo coordinado social, económico y educativo en América Latina. Bogotá, Colombia: Ediciones Alcaraván (p.21).

Banco Mundial. (2000). La educación superior en los países en desarrollo. Peligro y promesa (p.11). Recuperada el 29/01/13 de: www.tfhe. net/report/downloads/report/bm.pdf

Bourdieu, P. (2008). Homo academicus. Buenos Aires, Argentina: Siglo XXI Editores (p.171).

(2000). Las estructuras sociales de la economía. Barcelona, España: Editorial Anagrama (p.95).

Bourdieu, P. y Passeron Jean Claude. (2001). La reproducción. Elementos para una teoría del sistema de enseñanza. Madrid, España: Editorial Popular (p.34).

Bustamante, D. (1996). Misión nacional para la modernización de la universidad pública. Informe final. Bogotá, Colombia: Editorial Presencia (pp.17-70).

Coombs, P. (1971). La crisis mundial de la educación. Barcelona, España: Ediciones Península (p. 10).

Corredor, C. (1992). Los límites de la modernización. Citado por Zuluaga, J. (1992). Los límites de la modernización. Análisis Político №. 17. (sept./dic.). (pp.110-112).

Dávila, C. (1985) Teorías organizacionales y administración. Enfoque crítico. Citado por: Weiss, A. (1994). La empresa colombiana entre la tecnocracia y la participación: del Taylorismo a la Calidad Total. Bogotá, Colombia: Departamento de Sociología, Universidad Nacional de Colombia (p. 49).

Deleuze, G. (1987). Foucault. México. Paidós (p.34).

Díez, R. (1987). La educación ante las innovaciones científicas y tecnológicas. Citado por Castro, $M$. (2001). Dinámica del currículo y modernización de la universidad. Revista de la Universidad de la Salle, 22 (32) (pp. 36-37).

Escobar, A. (1996). La invención del tercer mundo. Construcción y deconstrucción del desarrollo. Bogotá, Colombia: Grupo Editorial Norma (p.13).
Foucault, M. (2005) Un diálogo sobre el poder y otras conversaciones. Madrid, España: Alianza Editorial (p.76).

(1976). Historia de la sexualidad, 1: La voluntad de saber. Citado por Díaz, M. (1993). El campo intelectual de la educación en Colombia. Cali, Colombia: Universidad del Valle (p. 213)

Giraldo, C. (2004). Ingeniería Industrial: Entre las ciencias exactas y las ciencias sociales. Documentos CESO № 72. Bogotá, Colombia: Ediciones Uniandes (p.8).

Hakim, R. (1981). La educación superior en Colombia a partir de la reforma de 1980. Desarrollo indo americano, 16 (67) (p. 8).

Henao, M. y Rivera, A. (1975), Universidad-Estado. Contribución para un estudio sobre la educación superior en Colombia. 1920-1940. Monografía para optar al título de sociólogas. Facultad de Sociología, Universidad Social Católica de la Salle, Bogotá, Colombia (pp. 57-59).

ICFES. (1996). Actualización y modernización del currículo en ingeniería industrial. Documento ejecutivo. Bogotá, Colombia: ACOFI, ICFES (pp.5-19).

Escobar, A. (2007). La invención del tercer mundo. Construcción y deconstrucción del desarrollo. (2007). Caracas, Venezuela: Ediciones el Perro y la Rana (p.109).

Martínez, A. (2004). De la escuela expansiva a la escuela competitiva. Dos modos de modernización en América Latina. Barcelona, España: Anthropos Editorial (p.49).

Mayor, A. (1984). Ética, trabajo y productividad en Antioquia: una interpretación sociológica sobre la influencia de la Escuela Nacional de Minas en la vida, costumbres e industrialización regionales. Bogotá, Colombia: Tercer Mundo Editores (p. 63).

Mejía, A. (2009). Tres esferas de acción del pensamiento crítico en ingeniería. Revista Iberoamericana de Educación, (49/3) (p 5-6). Recuperado 29/01/13 de: http://www.rieoei.org/ deloslectores/278oMejia.pdf

Melo, J. (1990). Algunas consideraciones globales sobre la modernización. Colombia el despertar de la modernidad. Recuperado el 15/03/11 en: http://www.jorgeorlandomelo. com/modernidad.htm 
Payán, S. (2001). Una reflexión sociológica sobre el trabajo. Trabajo XXI, Recuperado 21/05/10 en: http://www.galeon.com/grupogest/articulos/ artooo6.htm

Pérez, Y. (1990). Los orígenes de la ingeniería industrial en Colombia. Ingeniero Javeriano, (21) (p. 1025).

Pérez, Y. (2004). Presentación. Encuentro Nacional: La Ingeniería Industrial que Colombia Necesita para el Siglo XXI. Bogotá, Colombia, 19 y 20 de febrero. Universidad Javeriana (p. 7-8).

Pontificia Universidad Javeriana. (2010). Carrera Ingeniería Industrial. Reseña histórica. Recuperada 29/01/2013 de: http://puj-portal.javeriana. edu.co/portal/page/portal/Facultad\%20de $\% 20$ Ingenieria/plt_car_industrial/Rese $\% F_{10} \% 20$ hist\%F3rica

Poveda, G. (1985). La ingeniería, sus ciencias y su historia en Colombia: 1950 hasta nuestros días. Revista Universidad EAFIT, (58) (p.99).

Quintero, L. (2004). La profesión de la ingeniería industrial en Colombia. Encuentro nacional la ingeniería industrial que Colombia necesita para el siglo XX. Bogotá, Colombia, 19-20 de febrero. Universidad Javeriana (p. 13).

Savage, C.H, \& Lombard G.F. (1986). Sons of the machine. Case studies of social change in the workplace. Citado por Weiss, A. (1994). La empresa colombiana entre la tecnocracia y la participación: del Taylorismo a la Calidad Total. Bogotá, Colombia: Departamento de Sociología, Universidad Nacional de Colombia (p.39).

Touraine, A. (2000). Crítica de la modernidad. Bogotá, Colombia: Fondo de Cultura Económica (pp. 146)

(1969) La sociedad post-industrial. Barcelona, España: Ediciones Ariel (pp. 144-145).

UNESCO. (1998). Conferencia Mundial sobre la Educación Superior. La educación superior en el siglo XXI. Visión y acción. Informe Final (p. 11). Recuperado 28/01/2013 de: http://www.unesco. org/education/educprog/wche/declaration_spa. htm

Weiss, A. (1994). La empresa colombiana entre la tecnocracia y la participación: del Taylorismo a la Calidad Total. Bogotá, Colombia: Departamento de Sociología, Universidad Nacional de Colombia (p. 42). 